\title{
Performance Analysis of Wireless Location and Velocity Tracking of Digital Broadcast Signals Based on Extended Kalman Filter Algorithm
}

\author{
Yukai Hao ${ }^{1,2}$ and Xin Qiu $\mathbb{D}^{1}$ \\ ${ }^{1}$ School of Management, Wuhan University of Technology, Wuhan 400070, China \\ ${ }^{2}$ School of Economics and Management, Tibet University, Lhasa 850000, China \\ Correspondence should be addressed to Xin Qiu; qiux@whut.edu.cn
}

Received 26 November 2020; Revised 16 January 2021; Accepted 25 January 2021; Published 4 February 2021

Academic Editor: Wei Wang

Copyright (c) 2021 Yukai Hao and Xin Qiu. This is an open access article distributed under the Creative Commons Attribution License, which permits unrestricted use, distribution, and reproduction in any medium, provided the original work is properly cited.

In order to improve the accuracy and reliability of wireless location in NLOS environment, a wireless location algorithm based on artificial neural network (ANN) is proposed for NLOS positioning error caused by non-line-of-sight (NLOS) propagation, such as occlusion and signal reflection. The mapping relationship between TOA and TDOA measurement data and coordinates is established. The connection weights of neural network are estimated as the state variables of nonlinear dynamic system. The multilayer perceptron network is trained by the real-time neural network training algorithm based on extended Kalman (EKF). Combined with the statistical characteristics of NLOS error, the state component NLOS bias estimation is modified to realize TDOA data reconstruction. Simulation and experimental data analysis show that the algorithm can effectively weaken the influence of NLOS error. The localization method does not depend on the specific NLOS error distribution, nor does it need LOS and NLOS recognition. It can significantly improve the mobile positioning accuracy.

\section{Introduction}

With the development of social economy and the deepening of information process, people's demand for space information is becoming more and more urgent, and the satellite navigation technology is becoming more and more mature [1]. However, it is difficult to meet the requirements of precise positioning, due to the number of satellites and the geometric structure of satellites that can be observed in the high-rise urban area, so it is obviously unable to meet people's demand for location information only relying on satellite navigation. At present, the new generation of digital broadcasting system has covered most of the small- and medium-sized cities [2]. Because the terrestrial digital TV signal has many advantages, such as high transmission power, working in a lower frequency band, and being strictly synchronized with absolute time, the wireless passive positioning technology based on digital broadcasting signal is a positioning technology with good signal coverage and reasonable cost performance, especially strong antiattack ability, which can fill the market gap caused by the inherent deficiency of satellite positioning technology $[3,4]$.

The passive positioning system based on orbit satellite, such as GPS, has the huge advantages of wide coverage and providing global and all-weather navigation and positioning. Due to the multipath effect, GPS satellite orbit error, ionospheric delay effect, and so on, the positioning error of GPS technology is poor, so it takes $30 \mathrm{~s}$ or more to complete the initialization and satellite search work in cold start switching to normal navigation and positioning mode will delay the emergency rescue work [5]. Due to its perfect coverage and wide user groups, the wireless location system based on various terrestrial cellular networks naturally becomes one of the best radio location candidate technologies. However, since users have sent requests to obtain their own location, this active location mode is not conducive to the real-time and continuous positioning of users. In the process of electromagnetic wave propagation, multipath interference and nonline of sight error caused by obstacles and multiple 
access interference caused by "near far effect" in cellular communication system greatly affect the accuracy of mobile positioning, among which NLOS error is one of the most important factors. This system deduces the distance between the target and the TV tower according to the time of arrival of TV signal, but this distance value must be mixed with measurement noise and NLOS error [6]. NLOS error is the main source of wireless location error. Using NLOS toa to locate will bring great error to the result.

In view of the shortcomings and deficiencies of the above positioning system, this paper intends to adopt the wireless positioning algorithm of digital broadcasting signal based on artificial neural network. Through analysis of the characteristics of digital broadcasting signal, propagation characteristics, and the principle and method of wireless passive positioning system, this paper focuses on the research of high-resolution multipath time delay estimation and Doppler/micro-Doppler parameter estimation algorithm based on digital broadcasting signal, information fusion filtering method for mobile station real-time positioning and tracking using time delay and Doppler measurement information, and digital broadcasting single frequency network (SIN) Clustering analysis, data association, and fusion tracking algorithm of multisource data in the case of gel frequency network (SFN). The algorithm can effectively eliminate NLOS error, while locating the target, and make the positioning result more accurate. The range dynamic compensation is to predict the position of the target at the next moment according to the historical information, to achieve the purpose of dynamic compensation for the measured value and reduce the NLOS error. The subsequent linear line of position algorithm further eliminates the additive NLOS error by calculating the difference of the square of the distance. It directly uses the linear statistical positioning technology instead of simply linearizing the system and reduces the calculation amount of the target position, so it has certain advantages.

\section{Related Theories and Technologies}

\subsection{Principle of Digital Broadcasting Positioning System.} The broadcasting base station in digital broadcasting is the basic unit of terrestrial broadcasting supplementary system. It broadcasts mobile multimedia data and undertakes the task of transmitting spread spectrum positioning signal $[7,8]$. The ground broadcasting base station adopts the single frequency network design networking; each base station equipment uniformly receives and forwards the mobile broadcast signal from the medium orbit broadcast satellite. Each base station atomic clock is used as the reference clock, and the GPS multistation common view technology is used for accurate time correction, to ensure the accurate time synchronization accuracy of each base station in the SFN better than $15 \mathrm{~ns}$ [9].

Digital broadcasting positioning system is shown in Figure 1 . In the mobile broadcasting single frequency network, each mobile broadcasting base station broadcasts different and unique positioning spread spectrum code signals. The positioning signal adopts gold code with code length of 511, which is modulated in the initial taxed time slice of every 40 time slots of mobile broadcast data. When the base station continuously broadcasts the spread spectrum positioning pseudorandom code inserted in each time slot of the multimedia data, the mobile terminal can carry out channel estimation and pseudorandom code ranging and obtain the time synchronization correction information between the base stations through the time service message. The mobile terminal demodulates not less than $h$ spread spectrum positioning codes transmitted by broadcasting base stations, then the TDOA parameter measurement $W$ and location estimation can be completed [10]. The map display of terminal coordinates can be realized by combining the calculated positioning results with the terminal electronic ground fixation system. At the same time, the positioning results can also realize interactive location service through mobile communication network [11]. The positioning parameters and results are also transmitted back to MPC and PDE through mobile communication base station, BSC and mobile exchange. Relying on the GIS system of mobile communication network, LBS services such as positioning and navigation on the network side can be implemented.

The mobile terminal detects the initial phase and phase offset of spread spectrum codes from different base stations, calculates the delay difference of signal propagation, and obtains the TDOA parameters of signal propagation between two base stations. In the actual parameter measurement, the TDOA parameter of the signal between the primary base station of the cell and other adjacent base stations is selected as the basic parameter of the positioning solution [12]. The demodulation parameters include the signal strength $w$ of all the received base stations and the PN code offset relative to the positioning signal of the primary base station. Using the $\mathrm{PN}$ phase of the main base station as a reference, the TDOA measurement data can be obtained by measuring the code phase offset of PN code transmitted by adjacent base stations for positioning calculation. As long as the mobile station can detect and lock at least different PN code positioning signals from the base station, it can locate the mobile station. The composition of digital broadcasting positioning system is shown in Figure 2.

The positioning method in this paper is based on the radio signal broadcasting transmission system:

(1) Receiving signal: the TV signal received by the antenna is converted into IF signal after RF amplification.

(2) Through analog/digital (A/D) conversion, if signal digitization is completed.

(3) The synchronous tracking, demodulation, and decoding of signals are completed in the digital signal processor (DSP).

(4) The time interval of broadcasting data frame in DTV system is regarded as the basic time unit of system observation, and the observation time series is determined by the data frame interval or its multiple as the observation interval.

(5) The original pseudorange observation value of a digital TV transmitter is extracted from the 


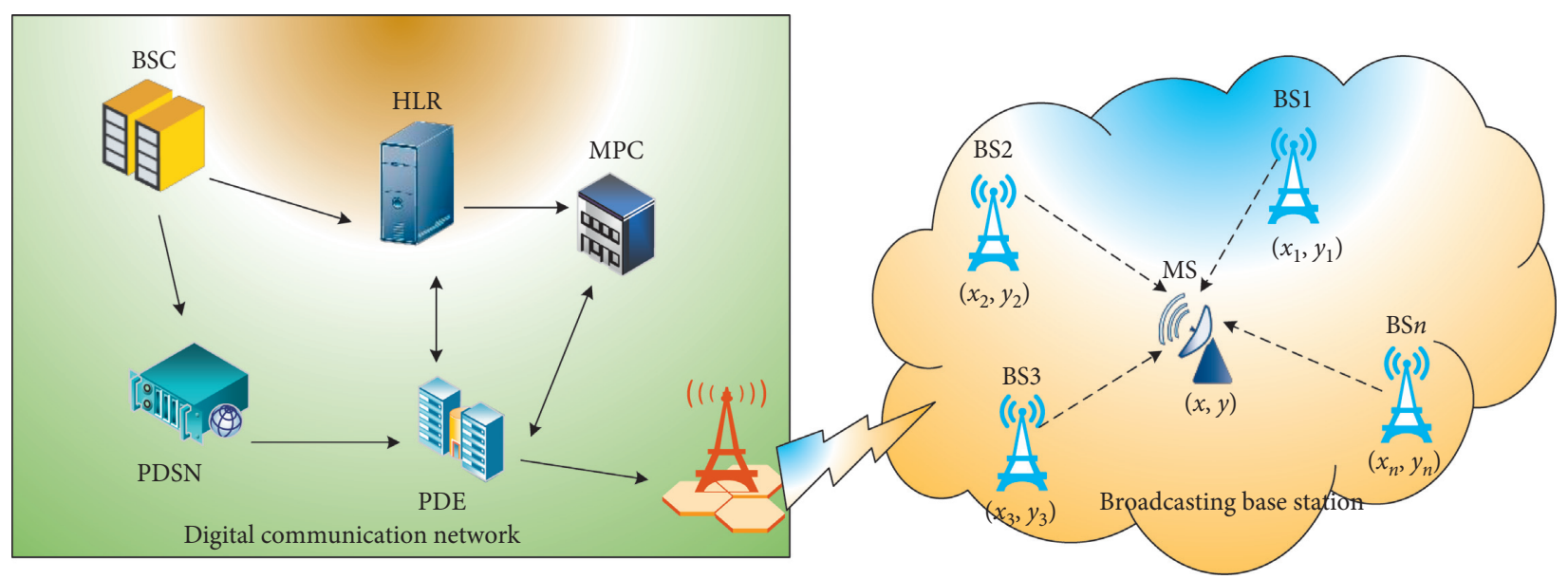

FIgURE 1: Positioning system based on digital broadcasting network.

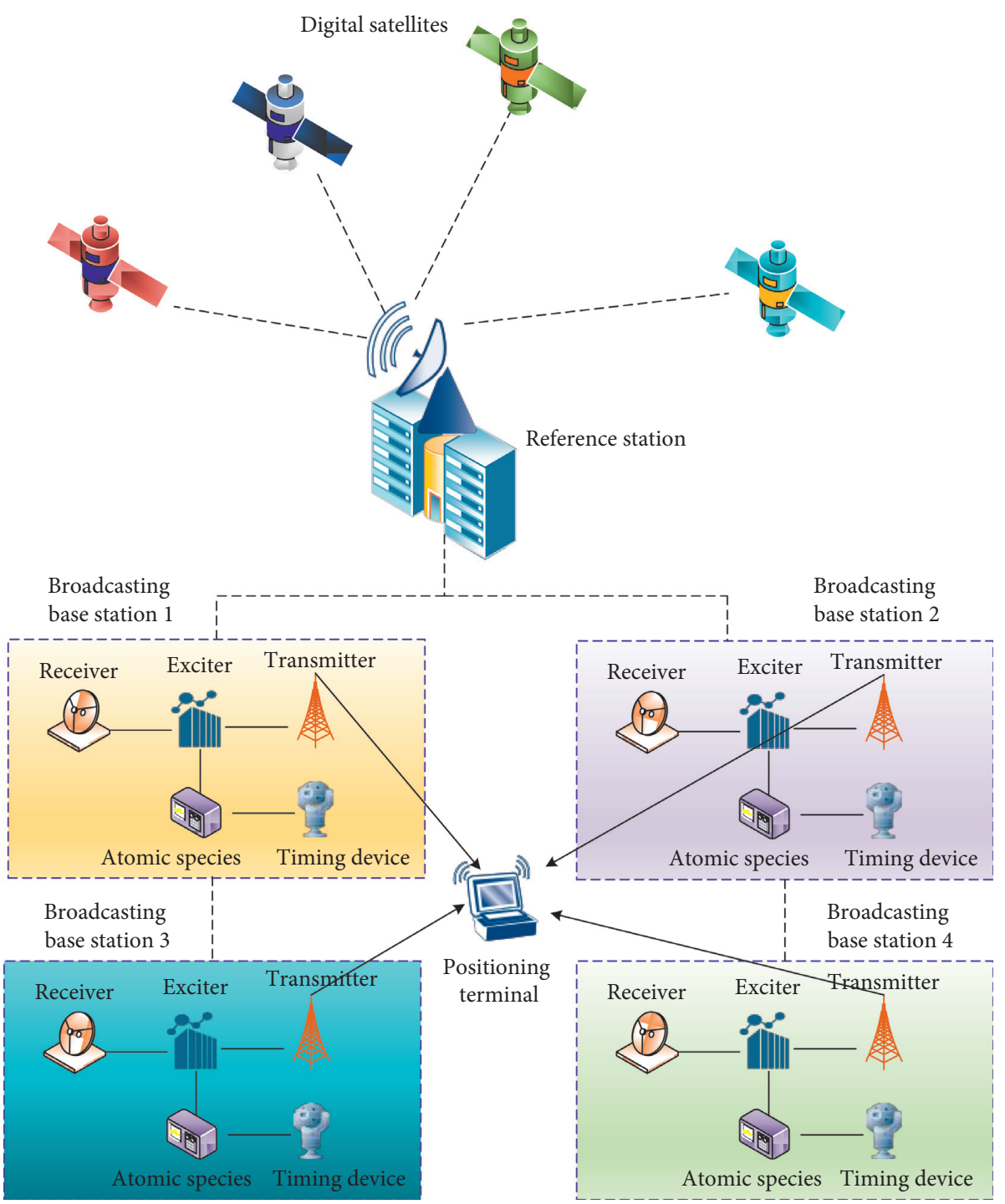

FigURE 2: Composition of digital broadcasting positioning system. 
synchronous tracking loop according to the time timing of the prescheduled observation sequence.

(6) Repeat step (5), and observe and track all digital TV transmitting stations within the effective measurement range; send all the obtained information into the pseudorange solution equation; that is, extract the pseudorange value and space coordinate value of each transmitter from the receiving tracking loop, calculate the spatial position coordinates of the final receiving skyline, and convert them into the positioning information of the receiving system, including position and velocity and acceleration, which are basic navigation and positioning information;

(7) The receiving time of each subsequent DTB data frame is measured to obtain the pseudorange information of all effective synchronization frame heads at the observation time point [13].

(8) The basic navigation and positioning information obtained in step (6) is substituted into pseudorange information and receiving time information in step (7), and the calculation based on sequential double filter smoothing algorithm is completed, and the optimal positioning information of the system is finally output. For DVB-T standard, the synchronization part uses the time domain protection interval and frequency domain pilot signal to estimate and track the position of FFT window in time domain and estimate the frequency offset caused by updown frequency conversion at the receiver and transmitter; sampling clock synchronization estimates the sampling clock error caused by the mismatch between the receiving and receiving crystal oscillators and synchronizes the receiving and receiving sampling clock through digital phase-locked loop. For DMB-T standard, $\mathrm{CP}$ in the traditional DVB-T system is replaced by a PN sequence, and no pilot is inserted into IDFT frame body. PN frame header is not only used as a training sequence for synchronization and channel estimation, but also used for protection interval objectively [14]. Each frame of DMB-T adopts different PN header as a frame mark, BPSK modulation is used by the PN header at transmitter to obtain reliable transmission effect; at the receiver, local PN sequence is generated by the same PN sequence generator, and $\mathrm{PN}$ of received signal is generated. The frame header is correlated in time domain to complete a series of synchronization operations such as frame synchronization, frequency synchronization, time synchronization, and channel transmission characteristic estimation [15].

2.2. Analysis of Positioning Mathematical Model. Geometric location method calculates the location information of unknown target by measuring the distance between the known reference point and the unknown target, which is the most widely used location algorithm. The common ranging methods include time of arrival (TOA), time difference of arrival (TDOA), and received signal strength (RSS).

2.2.1. Time of Arrival. The principle is to measure the time when the signals from three or more base stations arrive at the terminal under the condition of known radio propagation speed, then the distance between the receiving terminal and the base station can be uniquely determined, as shown in Figure 3.

The distances $R_{1}, R_{2}$, and $R_{3}$ between $\mathrm{MS}$ and $\mathrm{BS}_{1}, \mathrm{BS}_{2}$, and $\mathrm{BS}_{3}$ were measured, respectively. Then, MS is on the circle with BS coordinates as the center and the distance between MS and BS as the radius. Therefore, it forms three circles with three BS, and the terminal position is the intersection point of the three circles. The equations are shown as follows:

$$
\sqrt{\left(\lambda_{i}-\lambda_{0}\right)^{2}+\left(\gamma_{i}-\gamma_{0}\right)^{2}} \varepsilon_{i}, \quad i=1,2,3,
$$

where $\left(\lambda_{i}, \gamma_{i}\right)$ is the known BS coordinate, $\left(\lambda_{0}, \gamma_{0}\right)$ is the terminal coordinate, and $d$ is the distance between the terminal and the $i$-th base station. However, this method requires very high clock synchronization between the base station and the receiving terminal (one US clock synchronization error will lead to a distance error of 300 meters). Therefore, the cost of receiving terminal is greatly increased, and sometimes time synchronization cannot be achieved. In this paper, the least square method (LS) is used to solve the nonlinear equations to obtain the MS coordinates.

$$
H=\left[\begin{array}{cc}
\lambda_{2}-\lambda_{1} & \gamma_{2}-\gamma_{1} \\
\lambda_{3}-\lambda_{1} & \gamma_{3}-\gamma_{1} \\
\cdots & \cdots \\
\lambda_{n}-\lambda_{1} & \gamma_{n}-\gamma_{1}
\end{array}\right] .
$$

2.2.2. Time Difference of Arrival. Measure the time difference between two or more groups of two base stations arriving at the terminal, and use this time difference to calculate the terminal position. Compared with TOA method, this method does not require strict clock synchronization between the receiving terminal and base station; most land-based navigation systems use this method. The principle is shown in Figure 4.

Similar to the toa method, the $i$-th BS coordinate is marked as $\left(\lambda_{i}, \gamma_{i}\right)$, the MS coordinate is marked as $\left(\lambda_{0}, \gamma_{0}\right)$, the distance difference between $\mathrm{MS}$ and $\mathrm{BS}_{1}$ and $\mathrm{BS}_{2}$ is marked, the distance difference between $\mathrm{MS}$ and $\mathrm{BS}_{1}$ and $\mathrm{BS}_{3}$ is marked, and then the terminal position is the intersection point of two groups of hyperbolas. It can be obtained by solving the equations. In the case of multiple groups of solutions, solutions that do not conform to the actual position are easily excluded [16].

2.3. Artificial Neural Network. An MLP consists of several layers of nodes, which express artificial neural units. Each node, connected by the links with all the nodes in the 


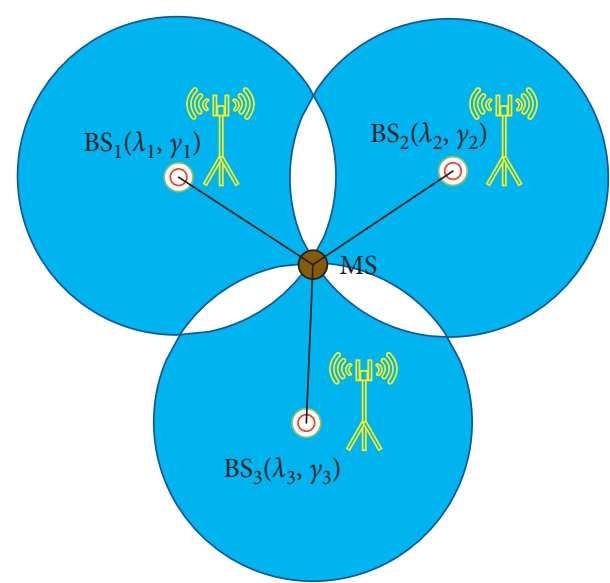

FIGURE 3: Positioning principle based on signal propagation time.

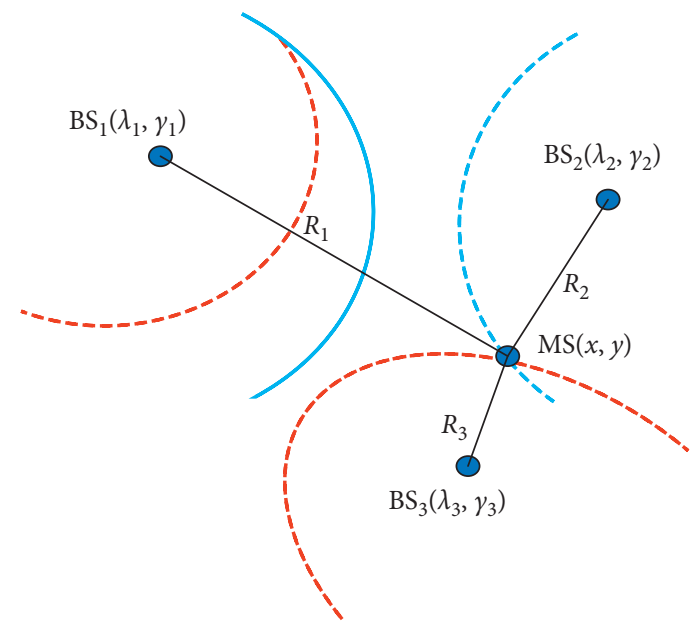

FIGURE 4: TDOA hyperbolic positioning.

adjacent layer, computes a weighted sum of inputs and then adds an offset to the sum. The computed result is the output through a nonlinear function. The three-layer structure MLP scheme for the wireless location is described. In this paper, the least number input measurements, three TOAs, and two TDOAs are employed to estimate MS location, and the network shown has only one hidden layer, which is based on the fact that the small size would be preferable to require a shorter time for both training and actual position estimations. The outputs of the coordinates of the corresponding position are shown in Figure 5.

Let the $i$-th node in the $n$-th layer be denoted by the node $(n, i) ; x^{\prime}(r)$ is the output of the node $(n, i)$ for pattern $t$, and $x$, the link weight from the node $(n, j)$ to the node $(n+1, j)$, and the offset of the node $(n, i)$ are expressed, and, respectively, each node in the input layer is assumed to perform no operation.

Furthermore, the offset is treated as the link weight by putting

$$
\lambda^{n}(t)=1, \quad 1 \leq n \leq s,
$$

where $n$ stands for the total number of nodes in the $n$-th layer, and $S$ represents the total number of layers including input

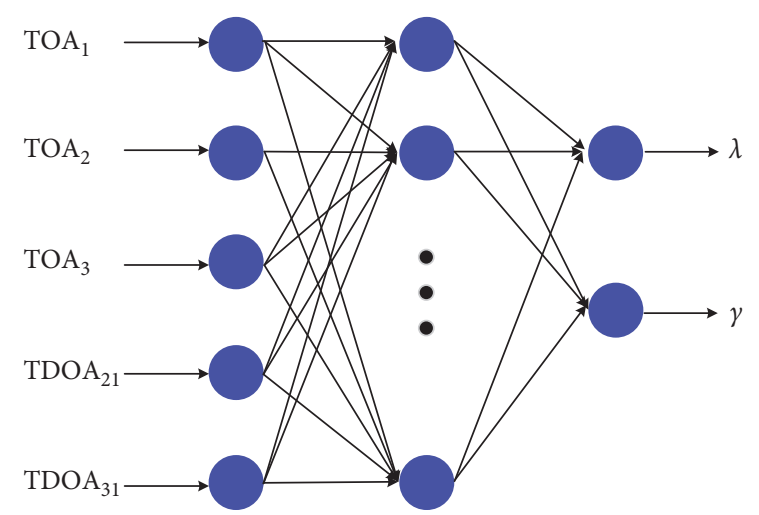

FIGURE 5: Structure of multilayered neural network for wireless positioning.

and output layers, and the operation of the node $(n+1, i)$ is then characterized.

The conventional BP algorithm iteratively adjusts the link weights using the SD technique so that the differences between the outputs of the MLP and the desired outputs are minimized. However, the convergence speed is slowly inherent, because the learning rate is fixed. Furthermore, we have to tune the learning rate and the momentum term in a heuristic manner so that a quick convergence is obtained $[17,18]$. An improper choice of these parameters may cause problems of unitability or suffer from much slower convergence. The advent of a more powerful learning algorithm instead of the SD-based algorithm has been expected. Using the EKF for updating the weights of an MLP can result in faster convergence in the sense that fewer training iterations are needed. Since the connection weight vector can be viewed as the state of a static nonlinear dynamic system, we set the unknown link weights as the state vector.

$$
\mu=\left[\left(\mu^{1}\right)^{T},\left(\mu^{2}\right)^{T}, \ldots,\left(\mu^{s-1}\right)^{T}\right]^{T}
$$

where

$$
\begin{aligned}
& \mu^{n}=\left[\left(\mu_{1}^{n}\right)^{T},\left(\mu_{2}^{n}\right)^{T}, \ldots,\left(\mu_{n-1}^{n}\right)^{T}\right]^{T}, \\
& \mu_{i}^{n}=\left[\mu_{i, 1}^{n}, \mu_{i, 2}^{n}, \ldots, \mu_{i, n}^{n}\right]^{T} .
\end{aligned}
$$

Let the output vector of the nodes in the $n$-th layer and the desired output vector of the mop be

$$
\begin{aligned}
\lambda^{n}(t) & =\left[\lambda_{1}^{n}(t), \lambda_{2}^{n}(t), \ldots, \lambda_{n}^{n}(t)\right]^{T}, \\
d(t) & =\left[d_{1}(t), d_{2}(t), \ldots, d_{n}(t)\right]^{T} .
\end{aligned}
$$

where $d(t)$ is the desired output of the $i$-th node in the output layer for pattern. The MLP is then expressed by the following nonlinear system equations:

$$
\begin{aligned}
\rho(t+1) & =\rho(t), \\
d(t) & =h(a(t))+v(t)=\lambda^{s}(t)+\gamma(t),
\end{aligned}
$$

where $r(n)$ is the output vector of the nodes in the output layer for pattern. The input to the MLP for pattern combined 
with the structure of the MLP is expressed by a nonlinear time-variant function $h$. The observation vector is represented by the desired output vector $d(t)$, and $v(t)$ is assumed to be a white noise vector with covariance matrix $[19,20]$.

The improved particle filter algorithm based on the extended Kalman filter bank important sampling has fast convergence speed and robust performance, and the positioning and speed tracking performance are obviously better than those of the EKF and classical particle filter methods. According to the motion characteristics of the target, the range measurement value is dynamically compensated, and then the residual NLOS error is further eliminated by using the LLOP algorithm. It can effectively improve the accuracy of digital radio positioning.

\section{Simulation Results and Performance Analysis}

3.1. Analysis of Mesh Feature Parameter Model. For the indoor positioning system using the civil SSI parameter grid as the only characteristic parameter, the RSSI variation at the same receiver can reach $10-15 \mathrm{dbm}$ due to the indoor multipath, shadow fading, diffraction, and other factors. The main reason is that the indoor receiving power depends not only on the propagation distance $D$, but also on the obstacles in the path. In order to analyze the fluctuation of RSSI parameters in grid positioning, a rectangular area of $30 \mathrm{~m} \times 30 \mathrm{~m}$ is selected and divided into $10 \times 10$ grids. AP is located at $(16.5 \mathrm{~m}, 19.5 \mathrm{~m})$ position, and the transmission power is $20 \mathrm{DBM}, N=3$ and $\sigma_{\xi}=3 \mathrm{~dB}$. The RSSI distribution of AP received by each grid node is shown in Figure 6. It can be seen that the RSSI variation can reach 10 DBM in a certain grid, so the accuracy and stability of positioning cannot be guaranteed by using the civilian SSI as the only characteristic parameter of the grid.

3.2. Analysis of NLOS Error Distribution. In the simulation, three visible GPS satellites are selected, and the TV Tower is selected as the launching station. The sampling interval is $1 \mathrm{~s}$, and the distribution model of NLOS error with uniform distribution in $[0,1300] \mathrm{m}$ is adopted. Each time the trajectory tracking and positioning are completed, a root mean square error is calculated, and 1000 independent simulations are carried out. The mean value of the 1000 root mean square errors is taken as the final root mean square error. The positioning deviation of different algorithms is shown in Table 1.

Figure 7 shows the influence of $\sigma$ on the positioning error when the NLOS error is uniformly distributed within $[0,1300]$ $\mathrm{m}$. It can be seen from the figure that if the NLOS error follows uniform distribution, the average positioning error is the minimum. Taking $\sigma=1.68$, the range dynamic compensation and linear position line joint algorithm, lop algorithm, and Taylor series expansion method are used to carry out 1000 independent positioning simulations. Figure 8 depicts the distribution of the absolute values of the east deviation and the north deviation when the above three positioning algorithms are used in 1000 independent simulations. It can be seen that

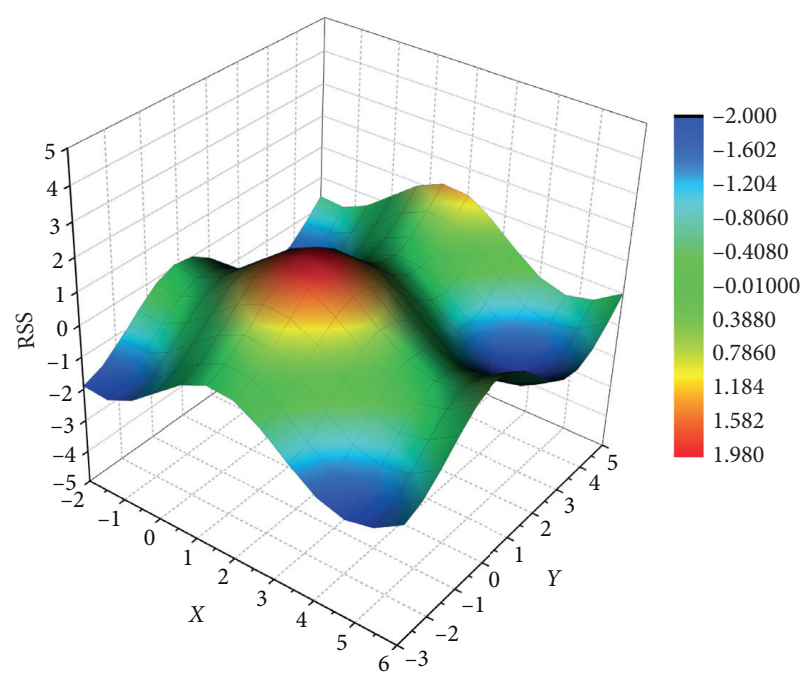

FIGURE 6: RSS distribution of grid nodes.

the deviation values of the joint algorithm are smaller than those of the other two traditional algorithms.

3.3. Trajectory Diagram for Positioning and Tracking at One Time. In the simulation experiment, the coordinates of the transmitting station are $[-3000 \mathrm{~m},-1000 \mathrm{~m}],[-3000 \mathrm{~m}$, $5000 \mathrm{~m}$ ], [6000 $\mathrm{m},-1000 \mathrm{~m}]$, and the real initial position of the mobile station is $[-1500 \mathrm{~m}, 1500 \mathrm{~m}]$, and it is assumed that the mobile station can always receive the signals from the above three transmitters during the motion. The movement state is a uniform motion with random acceleration, in which the transverse and longitudinal velocities are $50 \mathrm{~m} / \mathrm{s}$ and $0 \mathrm{~m} / \mathrm{s}$, respectively, and the army receives zero mean value. There is an influence of Gaussian random acceleration with variance of $0.52 \mathrm{~m} / \mathrm{s}^{2}$. The standard deviation of measurement noise is $150 \mathrm{~m}$, and the sampling interval $t=0.5 \mathrm{~s}$. In the comparison of algorithms, the number of particles in Sir algorithm is $n=5000$, and the number of particles in the new algorithm is greatly reduced. The initial positions of $N=500$. EKF and the mean value of initial position distribution of Sir and new algorithm are obtained by the algorithm. At the initial time, the prior velocity information of the mobile station is unknown. The mean values of the initial velocity of EKF and the particle velocity distribution in Sir and the new algorithm are set to $[0 \mathrm{~m} / \mathrm{s}, 0 \mathrm{~m} / \mathrm{s}]$.

Figure 9 shows the trajectory map of the three algorithms for positioning and tracking at one time. Obviously, for Sir algorithm, due to the large deviation between the initial mean velocity and the real velocity value, and the small random acceleration value in the state equation, the velocity cannot be effectively predicted and updated; even if 5000 particles are used, it cannot be tracked. Although EKF and the new algorithm can track, the new algorithm has fast convergence speed and small tracking error.

Figure 10 shows the position mean square error (RMSE) of EKF algorithm and the new algorithm through 20 times Monte Carlo simulation. Since Sir algorithm cannot track 
TABle 1: Positioning deviation of different algorithms.

\begin{tabular}{|c|c|c|c|}
\hline & $\mathrm{d} x(\mathrm{~m})$ & $\mathrm{d} y(\mathrm{~m})$ & Dis $(\mathrm{m})$ \\
\hline Wireless location algorithm based on artificial neural network & 3.947 & 6.537 & 7.987 \\
\hline LLOP & 5.612 & 9.824 & 11.536 \\
\hline Taylor & 9.883 & 10.138 & 15.387 \\
\hline $\mathrm{BP}$ & 8.568 & 6.384 & 10.235 \\
\hline
\end{tabular}

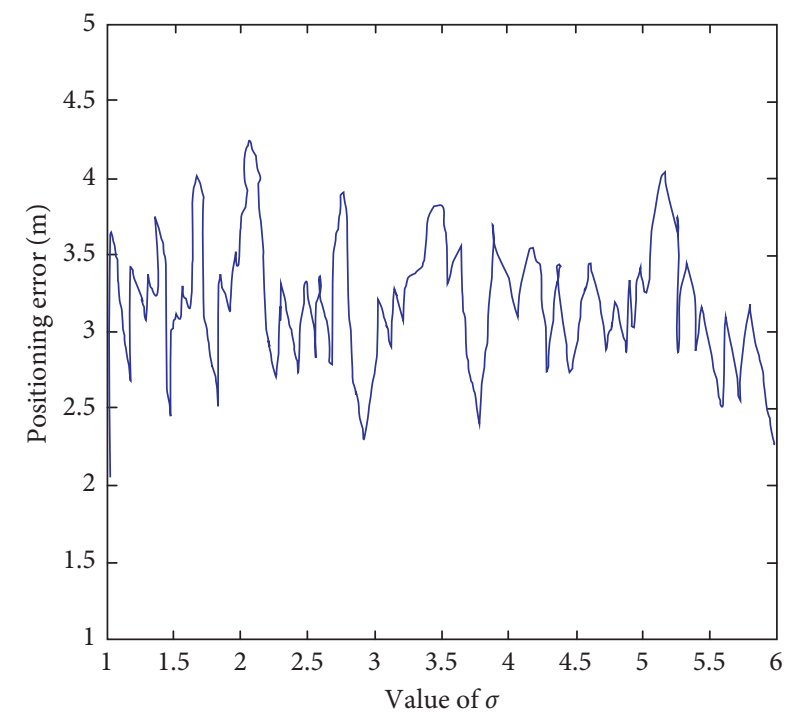

FIgURE 7: Relationship between value and positioning error.

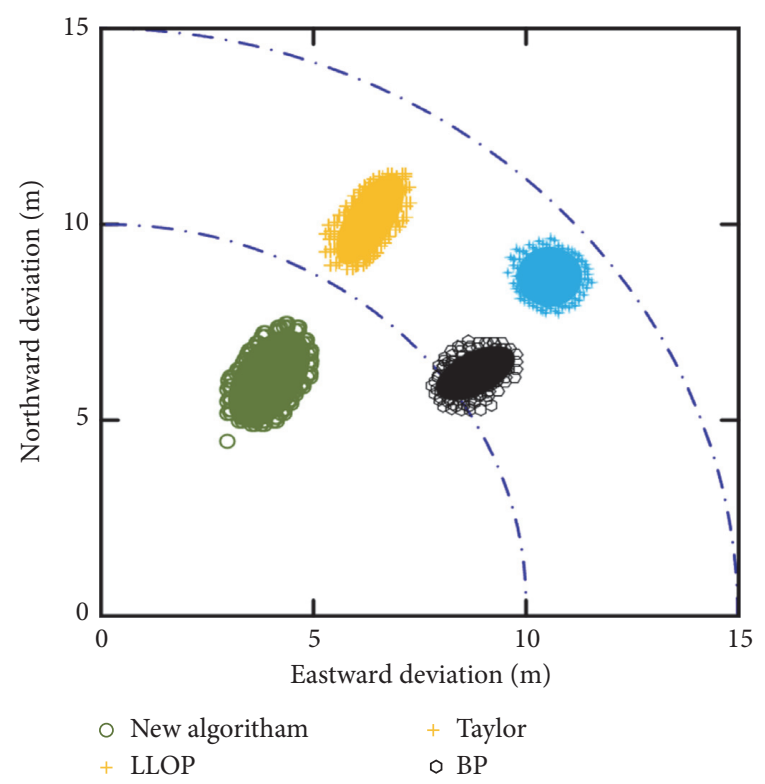

FIgURE 8: Deviation of different localization algorithms.

the mobile station correctly when the initial mean deviation is large, the algorithm is not compared. It can be seen from Figure 9 that, due to the large initialization error and certain error in the linearization process, EKF algorithm can track, but the error is large; after the time sampling point $k=30$ reaches the steady state, the total position RMSE is $54.5 \mathrm{~m}$,

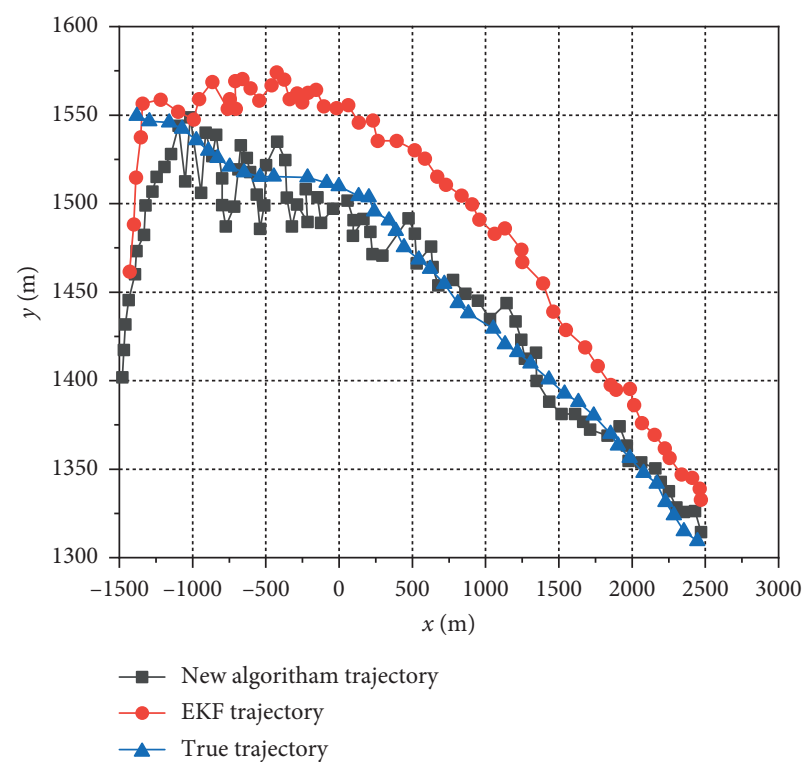

Figure 9: Comparison of real trajectory and one-time tracking results.

and the total speed RMSE is $6.4 \mathrm{~m} / \mathrm{s}$. The improved particle filter algorithm based on the important sampling function of EKF filter bank has great improvement in both position RMSE and velocity RMSE: at the time sampling point $k=3$, the position RMSE is reduced to $35.9 \mathrm{~m}$, the total position RMSE is $18 \mathrm{~m}$, and the total velocity RMSE is $4.3 \mathrm{~m} / \mathrm{s}$. The main reason for the performance improvement is that the influence of the current observation value is considered when constructing the important steps of the state vector, and the estimation of the speed is considered in the process of calculating the mean value and variance of each example separately. After resampling, the position estimation of the retained particles is relatively accurate, and the corresponding velocity error is also small.

Figure 11 shows the performance on root mean square error (RMSE) location error of three algorithms under different environments. All the RMSE location errors are obtained from the average of $L=1000$ independent runs with the same parameters. The figure contains four groups of bar plots, and each group corresponds to one of four environment types. Within a group, the RMSE values derived from the three algorithms are represented by the heights of three bars. Overall, the performance ranking from the worst to the best with respect to the environment types is as follows: bad urban, urban, suburban, and rural. As shown in Figure 11, the RMSE values of the proposed algorithm are smaller than the results of the LS and the BP algorithms in all of the scenarios, which convinces us that the proposed 


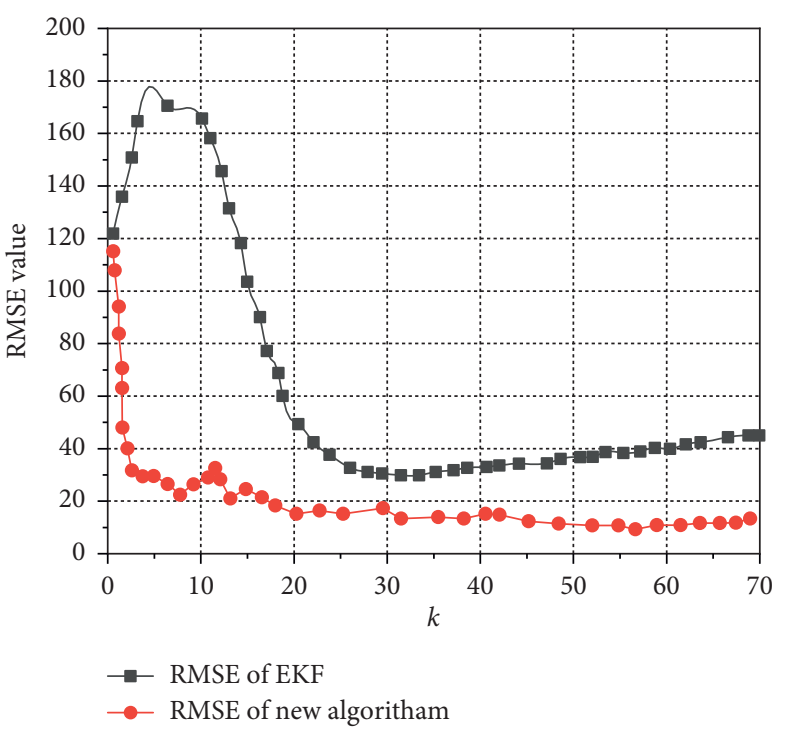

(a)

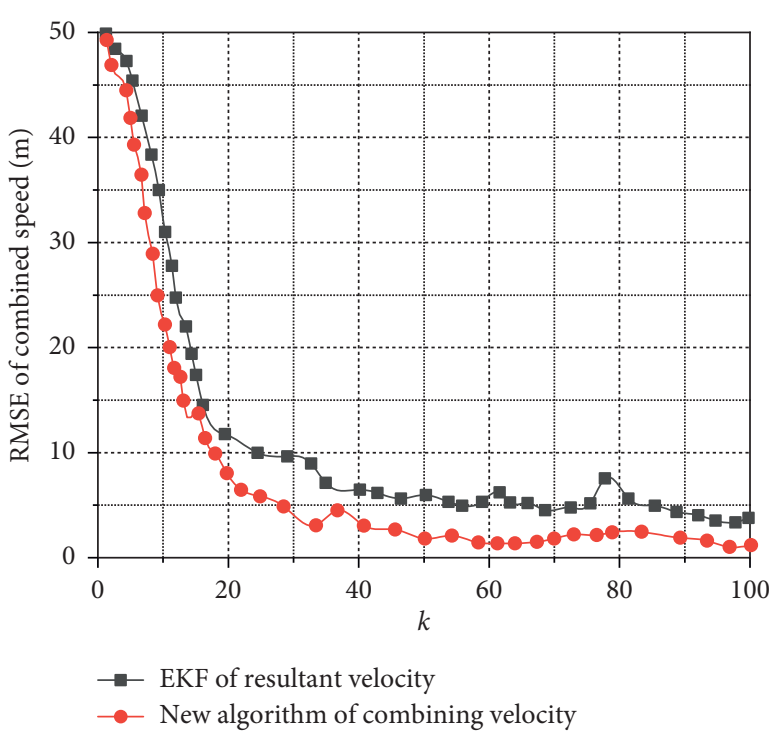

(b)

Figure 10: Comparison of position RMSE and velocity RMSE in different algorithms. (a) RMSE of location. (b) RMSE of velocity.

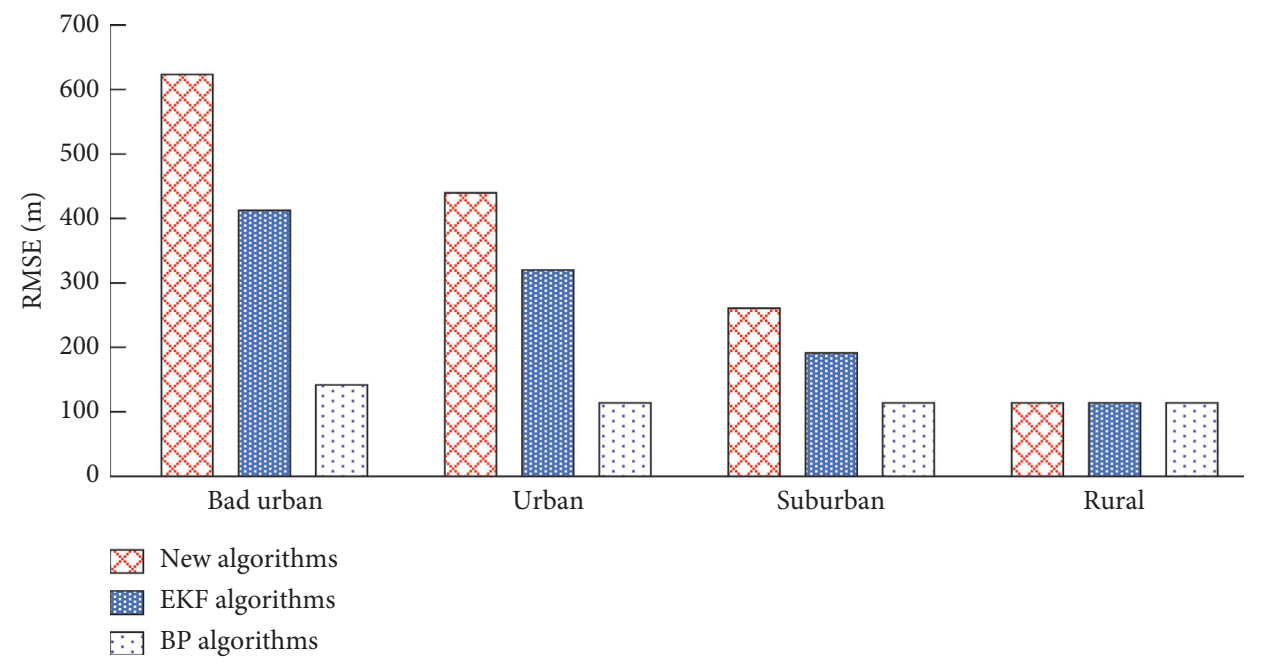

FIGURE 11: RMSE location error of different algorithms under different environments.

method can adapt well to real environments and gives a good estimation of the location of the MS. Compared with the BPbased and the LS-based algorithms, the RMSE of location errors is improved approximately from 200 to $350 \mathrm{~m}$ in an urban environment, and in a bad urban environment, the improvement even goes up to about 300 to $500 \mathrm{~m}$. For a suburban environment, the improvement is around 50 to $150 \mathrm{~m}$ : as for a rural environment, in which NLOS errors are not severe and do not leave much space for improvement, the performance improvement of the RMSE value is not very visible.

With the development of technology, terrestrial broadcasting, as a new information transmission technology, has begun to be widely used, and the wireless system based on this technology has also begun to build on a large scale, and a new passive positioning technology based on broadcast signal has also begun to be proposed and has attracted more and more attention of researchers at home and abroad [21, 22]. There are many advantages and potential advantages in wireless passive location using broadcast signal. Because of the wide application prospect of OFDM technology in many countries and abroad, OFDM technology has been widely used. According to the multipath propagation characteristics of digital terrestrial broadcasting, the micro-Doppler effect caused by the nonuniform motion of the mobile station relative to the remote transmitter in the positioning system is analyzed $[23,24]$. The micro-Doppler characteristics of the received signal of pilot subcarriers in the system are detected by FRFT, and the micro-Doppler parameters of each path are estimated by multicomponent LFM signal separation method. It has good estimation accuracy and good practicability. Although this paper has made a preliminary exploration on parameter measurement based on broadcast 
signal, information fusion filtering algorithm, clustering, association, and fusion tracking of multisource data in SFN environment and achieved encouraging results, positioning, especially in SFN environment, is a new topic, and there are still many areas to be improved and further studied [25]. The proposed OFDM time delay estimation method can accurately detect multipath signals and extract the first path toa information in NLOS multipath environment, so as to reduce the estimation error and improve the positioning accuracy. However, this method cannot eliminate the additional delay system error caused by NLOS propagation itself [26-28].

\section{Conclusion}

Wireless location system based on digital broadcasting is a new wireless positioning technology, in the process of positioning and tracking, and this paper proposes a wireless positioning algorithm based on artificial neural network for digital broadcasting signal, and particle filter improvement algorithm with important sampling of extended Kalman filter bank has fast convergence speed and stable performance. When the initial position and speed deviation are large, the algorithm has good performance, the performance of localization and velocity tracking is better than that of EKF and classical particle filter. This method can also be used for location based on other information such as angle of arrival and received signal strength. The algorithm can effectively weaken the NLOS error and can still achieve reliable positioning in the urban environment with many obstacles. The new algorithm dynamically compensates the range measurement value according to the motion characteristics of the target and then uses the lop algorithm to further eliminate the residual NLOS error. The theoretical analysis and simulation results show that the performance of the algorithm is better than the traditional Taylor series expansion method and can improve the positioning accuracy of the auxiliary navigation satellite signal hybrid positioning system in the harsh channel environment.

\section{Data Availability}

The data used to support the findings of this study are available from the corresponding author upon request.

\section{Conflicts of Interest}

The authors declare that they have no known conflicts of interest or personal relationships that could influence the work reported in this paper.

\section{Acknowledgments}

This work was supported by the Project Source Ministry of Science and Technology of the People's Republic of China (project number 2018YFB0804300).

\section{References}

[1] M. Yang, H. Wu, and Z. Liu, "Indoor positioning using public FM and DTMB signals based on compressive sensing," China Communications, vol. 16, no. 5, pp. 171-180, 2019.

[2] S. N. Daskalakis, J. Kimionis, A. Collado, G. Goussetis, M. M. Tentzeris, and A. Georgiadis, "Ambient backscatterers using FM broadcasting for low cost and low power wireless applications," IEEE Transactions on Microwave Theory and Techniques, vol. 65, no. 12, pp. 5251-5262, 2017.

[3] C. Y. Chen, L. X. Jiang, and J. S. Han, "Discussion on positioning method with digital FM broadcasting signals," Journal of Navigation and Positioning, vol. 5, no. 3, pp. 33-37, 2017.

[4] S. Takahashi, "Methods of receiving and broadcasting emergency warning signals for ISDB-T terrestrial digital television standard," Journal of Announce \& Nanotechnology, vol. 16, no. 3, pp. 3077-3083, 2014.

[5] H. Wang, L. N. Hong, and J. X. Yi, "A novel migration compensation algorithm for passive radar using digital TV signals," Journal of Electronics \& Information Technology, vol. 37, no. 5, pp. 1017-1022, 2015.

[6] F. Fava, M. Cantu, A. De Luca, and S. Hristov, "Passive multifrequency forward-scatter radar measurements of airborne targets using broadcasting signals," IEEE Transactions on Aerospace \& Electronics Systems, vol. 12, no. 7, pp. 10671087, 2019.

[7] E. Parasystole and A. A. Economides, "A novel anomaly detection and location-attribution algorithm for UWB wireless sensor networks," Journal on Information Security, vol. 14, no. 1, pp. 3-8, 2014.

[8] S. Wu, D. Xu, and H. Wang, "Adaptive NLOS mitigation location algorithm in wireless cellular network," Wireless Personal Communications, vol. 84, no. 4, pp. 3143-3156, 2015.

[9] X. Hua, Z. Jinjin, and B. Lei, "A new three-dimension spatial location algorithm of wireless sensor network," International Journal on Smart Sensing and Intelligent Systems, vol. 9, no. 1, pp. 233-255, 2016.

[10] W. Hua, W. Tingyu, and Z. Daze, "Location verification algorithm of wearable sensors for wireless body area networks," Technology and Health Care, vol. 26, no. 5, pp. 3-18, 2018.

[11] H. B. Wang, L. P. Feng, J. F. Cao, and C. Liu, "Wireless sensor network accurate TOA location algorithm based on the energy detection," International Journal of Wireless and Mobile Computing, vol. 10, no. 2, pp. 159-163, 2016.

[12] W. Jinghui, "A node location algorithm in wireless sensor network based on DV-Hop," Computer Engineering, vol. 41, no. 1, pp. 82-86, 2015.

[13] L. Rong and R. D. Dubicki, "Fuzzy weighted location algorithm for abnormal target in wireless sensor networks," Journal of Intelligent and Fuzzy Systems, vol. 35, no. 8, pp. 1-9, 2018.

[14] S. X. Lin, H. Z. Liu, and S. J. Chen, "Optimization and implementation of Zigbee wireless location algorithm," Revest de la Faulted de Ingenerate, vol. 32, no. 5, pp. 396-404, 2017.

[15] L. Song, L. Zhao, and J. Ye, "DV-hop node location algorithm based on GSO in wireless sensor networks," Journal of Sensors, vol. 2019, Article ID 2986954, 9 pages, 2019.

[16] X. Li, K. Wang, and B. Liu, "An improved range-free location algorithm for industrial wireless sensor networks," Journal on 
Wireless Communications and Networking, vol. 20, no. 1, pp. $178-185,2020$.

[17] B. Xu, S. Xiang, and X. Wu, "The wireless sensor network location algorithm based on three-dimensional spherical segmentation," Chinese Journal of Sensors and Actuators, vol. 31, no. 9, pp. 1419-1425, 2018.

[18] G. M. Kombi and O. P. Kogod, "Gateway location algorithm for wireless mesh networks," Journal of Engineering and Applied Fences, vol. 13, no. 17, pp. 7288-7297, 2018.

[19] C. M. Wu, X. Yang, and H. Q. Gong, "Node tacking location algorithm for wireless sensor networks based on improved particle filter," Journal of Information Hiding and Multimedia Signal Processing, vol. 9, no. 4, pp. 785-795, 2018.

[20] Z. Hu, K. Zhang, and Z. Wang, "The node location algorithm for wireless sensor networks based on saddle topography," Chinese Journal of Sensors and Actuators, vol. 31, no. 11, pp. 1753-1757, 2018.

[21] B. Xiang, "Application of ranging difference location algorithm in wireless sensor network location," International Journal of Online Engineering (IJOE), vol. 13, no. 7, pp. 81-85, 2017.

[22] W. Han, S. Li, and W. Li, "Study of wireless sensor network location algorithm and data collection protocol," International Journal of Online Engineering (IJOE), vol. 12, no. 10, pp. 70-78, 2016.

[23] E. S. Taliedo, F. H. Awed, and S. M. Abdullah, "Transitive location verification algorithm in location-based routing for wireless sensor networks," Ad Hoc \& Sensor Wireless Networks, vol. 21, no. 3-4, pp. 237-257, 2014.

[24] S. Ruchi, C. T. Jong, and A. C. Wok, "A genetic algorithm with location intelligence method for energy optimization in $5 \mathrm{G}$ wireless networks," Discrete Dynamics in Nature and Society, vol. 2016, Article ID 5348203, 9 pages, 2016.

[25] I. Baskaran and A. Albeola, "A new distributed location-based routing algorithm for wireless sensor networks," Cybernetics and Systems, vol. 45, no. 7, pp. 599-616, 2014.

[26] M. Zhou, Y. Wang, Y. Liu, and Z. Tian, "An information-theoretic view of WLAN localization error bound in GPS-denied environment," IEEE Transactions on Vehicular Technology, vol. 68, no. 4, pp. 4089-4093, 2019.

[27] W. Ke and L. N. Wu, "Direct non-line-of-sight bias estimation for positioning in digital broadcasting system," in Proceedings of the Asia Pacific Conference on Postgraduate Research in Microelectronics and Electronics (PrimeAsia), pp. 57-60, Shanghai, China, November 2009.

[28] L. Chen, H. Z. Zou, and L. N. Wu, "Modified particle filtering for wireless tracking in digital broadcasting system," in Proceedings of the International Conference on Wireless Communications, Networking and Mobile Computing, pp. 1-4, Dalian, China, October 2008. 